\title{
CONSERVATIVE GAP ARTHROPLASTY WITHOUT INTERPOSITIONING MATERIAL FOR MANAGEMENT OF TEMPOROMANDIBULAR JOINT ANKYLOSIS IN CHILDREN: CLINICAL AND RADIOGRAPHIC OUTCOMES IN UPPER EGYPT
}

\author{
Ahmed T. Temerek ${ }^{*}$ and Ali Fahd ${ }^{* *}$
}

\begin{abstract}
Ankylosis is one of the challenging disorders of the TMJ especially in children. Trauma, infection, and systemic diseases are the most common causes, factors common to occur in children in Upper Egypt. Unfortunately, TMJ ankylosis responds only to surgical intervention.

The aim of this study was to evaluate a conservative surgical approach.

Materials and Methods: In this study inclusion criteria were set and 11 children suffering from ankylosis enrolled for the most conservative gap arthroplasty without interpositioning material and with postoperative physiotherapy protocol. The patients were evaluated clinically and radiographically for 24 months postoperatively regarding mouth opening and recurrence.
\end{abstract}

Results: trauma was the main etiologic factor and bony ankylosis accounted for $81 \%$, the mean gain in mouth opening was $29 \mathrm{~mm}$ with no reported recurrence.

Conclusion: using conservative gap arthroplasty in children is a success option within the justified inclusion criteria.

\section{INTRODUCTION}

Temporomandibular joint (TMJ) ankylosis can be defined as fibrous or bony adhesion between the mandibular condyle and the glenoid fossa. Histologically, it can occur as fibrous, bony or fibroosseous adhesions between the mandibular condyle and the glenoid fossa ${ }^{[1,2]}$. The most common causes of TMJ ankylosis are trauma and infection ${ }^{[3-5]}$.
Untreated ankylosis especially during the growth bearing period leads to varying degrees of not only mandibular but also the whole maxillofacial skeleton deformities plus physical and psychological consequences. The complications associated with TMJ ankylosis are not only the limited mouth opening and loss of function but also the problems associated with facial growth and aesthetics ${ }^{[6,7]}$.

\footnotetext{
* Lecturer, Oral and Maxillofacial Surgery Department, Faculty of Oral and Dental Medicine, South Valley University, Qena, Egypt. ** Lecturer, Oral and Maxillofacial Radiology Department, Faculty of Oral and Dental Medicine, South Valley University, Qena, Egypt.
} 
Unfortunately, TMJ ankylosis responds only to surgical intervention combined with early aggressive physiotherapy protocols. Frequently used surgical protocols include: gap arthroplasty, interpositional arthroplasty or joint reconstruction with or without mandibular distraction for correction of the residual deformity. Many authors recommend creating an aggressive gap of $15-20 \mathrm{~mm}$ to evade relapse ${ }^{[5,8]}$. Multiple drawbacks were noted after wide gap creation. Recently different authors reported a comparable success rates using a more conservative management through a minimal gap measuring 5 to $8 \mathrm{~mm}$ with interpositional material ${ }^{[9]}$

The aim of this study was to evaluate the success rate of conservative gap arthroplasty without interpositioning material in the treatment of TMJ ankylosis in children.

\section{PATIENTS AND METHODS}

13 patients were recruited in the study (only 11 patients have been shown on the assigned follow up time and included in the results). They were diagnosed of TMJ ankylosis at the Out-patient clinic of the Oral and Maxillofacial Surgery Department, Faculty of Oral and Dental Medicine, South Valley University, Qena, Upper Egypt. Ankylosis was treated by conservative gap arthroplasty and no interposition material was used followed by immediate postoperative physiotherapy protocol. The study was preapproved by the research and ethics committee at South Valley University, and all patients agreed upon the informed consent.

\section{Inclusion and exclusion criteria:}

- Radiographic evidence of true ankylosis of the TMJ and not involving the sigmoid notch

- Patient age: 12 years old or less at the day of diagnosis

- Patient had no previous treatment for ankylosis
- Patient had no local or systemic contraindication for operation

- Patient completed the follow up protocol of at least 24 months

All patients were assessed from their history, clinical and radiographic examinations. Computed tomograms were used preoperatively to confirm the diagnosis, to assess the anatomical relations and for surgical planning (Fig. 1).

A data sheet (Table 1) was filled in for each patient to record age, sex, involved side, duration, etiology, type of ankylosis, maximum mouth opening before and after operation, follow-up period, recurrence and involvement of the facial nerve.

Patients were operated under general anaesthesia. After induction of anaesthesia, application of antiseptic, and standard surgical draping the TMJ was approached through an endaural incision (Fig. 2). The superficial temporal fascia is identified, and its plane followed inferiorly and anteriorly to reach the zygomatic arch and capsule of the TMJ. The ankylotic mass is exposed through an inverted-L incision, and the lateral bony prominence at the ankylotic mass is shaved to a flat ramus surface. If a cleavage line was found, it was used as the superior bony cut plane. If there was no cleavage line, an imaginary line through the lower border of the zygomatic arch is considered. A gap 7-9 mm wide was then created and measured with Castroviejo caliper with the mandible in a resting position. No interposition material was used. If the disc was present in good condition it is used to line the glenoid fossa at the gap. The transoral temporalis tendon and pterygomasseteric sling were released, the masseter reflected, and coronoidectomy done if the interincisal distance was less than $35 \mathrm{~mm}$.

Every patient had a custom-made acrylic bite block that was fitted to the occlusal surface of the molar-premolar segment after the desired mouth opening had been achieved and was subsequently 
removed from the mouth after all reflexes have been regained. Each acrylic bite block was left in place for the first three postoperative days and patients were restricted to a liquid diet. It was removed only at meal times during the next two weeks. Before and after each meal, patients were asked to move the chin upwards gently and gradually as much as they can to reach maximum intercuspation, and then keep it for 5 minutes. During the next three months the time for using the acrylic bite block was gradually reduced, and patients were encouraged to exercise intensively at home by opening and closing the mandible alternating with chewing three times a day for 15 minutes at a time. During the next six months, every patient was instructed to use the bite block at least twice weekly to check its fitness at home, and to contact the outpatient clinic at once in case of any difficulty or looseness of fit. Patients were seen twice weekly for six weeks at the outpatient clinic, and then followed up every three months for 24 months when the patients had their final radiographic evaluation (Fig.4,5). All patients were instructed for revaluation every six months for the next five years.

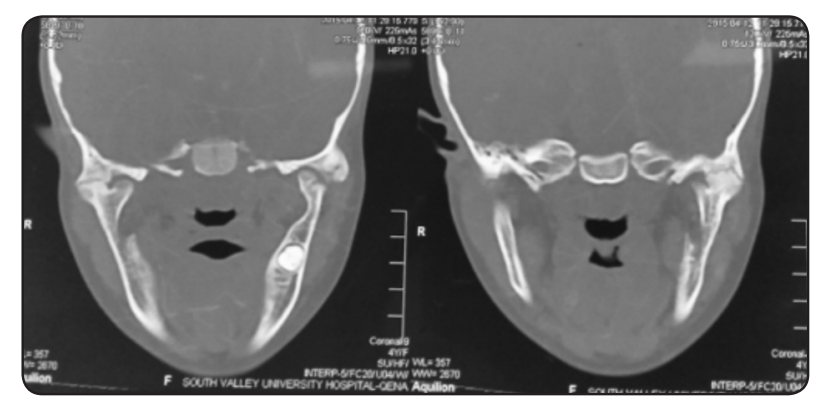

Fig. (1): Preoperative coronal CT images showing bilateral ankylosis and deformity in the joint. The bones are not totally fused but the two irregular surfaces are deformed and appear to fit one another like a jigsaw puzzle. Note the heart shaped condylar part (left).

\section{RESULTS}

Among 13 children patients aged 12 years or less and had true ankylosis managed during this interval, only 11 patients have fulfilled the inclusion criteria. All patients treated with a standard treatment protocol using conservative gap arthroplasty of $7-9 \mathrm{~mm}$ with no interpositional material and a protocol of physiotherapy that started from day 1 postoperatively.

Reported cases showed successful management of 11 patients (13 joints), aged from 4 to 12 years with a mean age of 7 years. Most of the reported cases were females comprising 63\%. Ankylosis was reported to occur at different intervals before operation ranging from 3 to 24 months. Trauma was the main etiologic factor accounting for $90 \%$ of the reported cases. Bony ankylosis as reported from preoperative $\mathrm{CT}$ and confirmed by the intraoperative notes accounted for $81 \%$. Disc was identified and used for lining the conservative gap in 3 cases. Mean reported preoperative mouth opening was 5 $\mathrm{mm}$ that increased to $33.8 \mathrm{~mm}$ at 2 years with no recurrence reported through the follow-up period. Facial nerve was affected temporarily in $18 \%$ of the cases and improvement occurred within 6 months postoperatively.

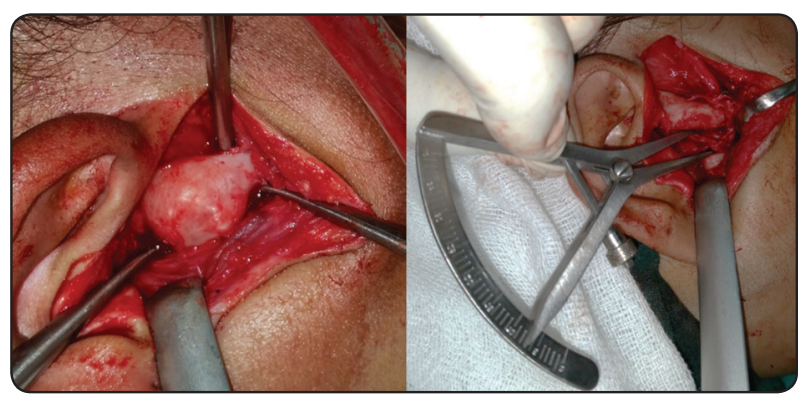

Fig. (2): Intraoperative clinical photograph showing the ankylotic mass before intervention (Left) and after creating a $7 \mathrm{~mm}$ gap measured with Castroviejo caliper with disc preservation (Right). 


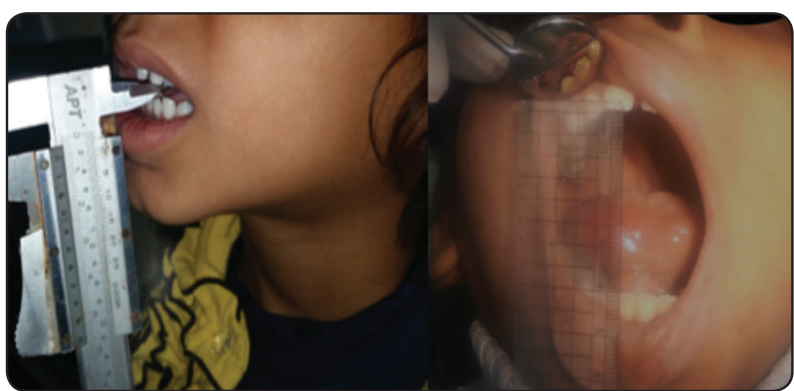

Fig. (3): Clinical photograph showing the mouth opening preoperatively (Left) and postoperatively (Right)

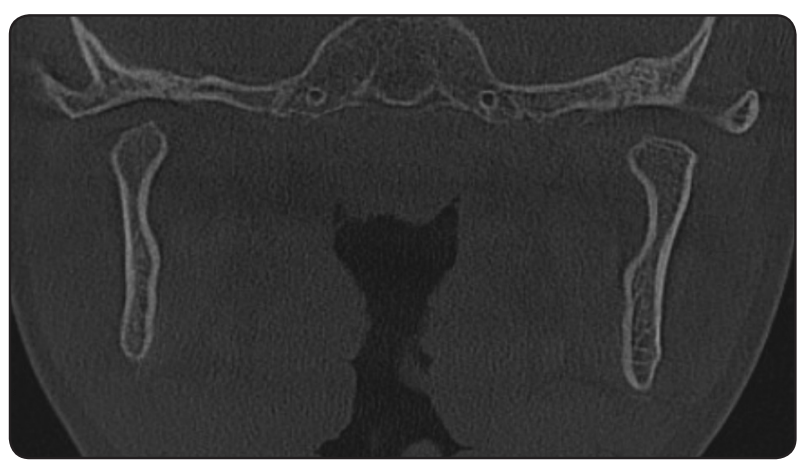

Fig. (4): Postoperative coronal CBCT image showing how the remaining parts are reshaped into a condyle like shape. Note the thin bone cortication at the articular surfaces.

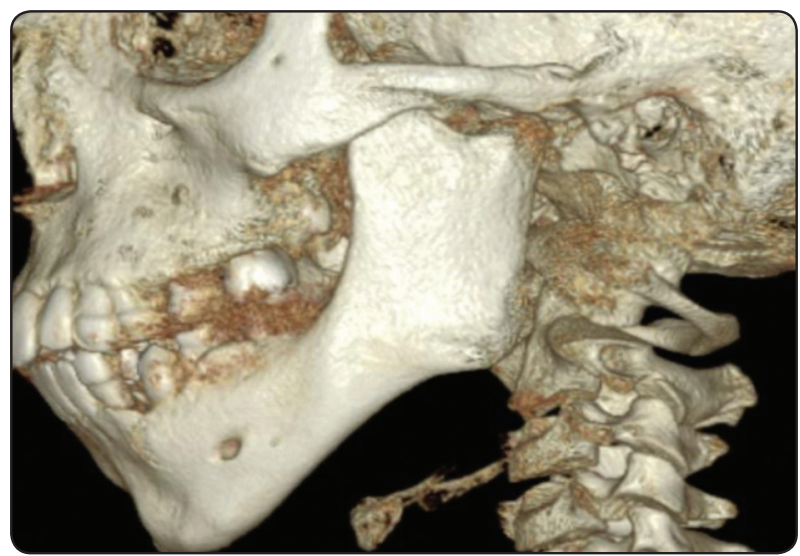

Fig. (5): Postoperative 3D reformatted view showing signs of remodeling and how the joint components adapt. Note the condyle like morphologic changes, the compensatory progressive elongation of the coronoid process and the deepening of the antegonial notch.

\section{DISCUSSION}

Temporomandibular joint ankylosis is an abnormal condition in which the condyle of the mandible is fused to the glenoid fossa either by bony or fibrotic tissues. In this study, all cases were diagnosed with true ankylosis based on clinical and radiographic examination. Injury or infection are the most common causes.

In childhood or growth bearing period, the clinical picture of ankylosis may be in the form of retrognathia, chin deviation, open bite and/or difficulty in respiration. These findings are mainly due to growth restriction and their magnitude differs depending on the age of occurrence, duration, being unilateral or bilateral and degree of movement restriction ${ }^{[10,11]}$. That was the motivation to do this study on this selected age group.

In the present cases, the prevalence of traumatic cause was $90 \%$. Posttraumatic joint ankylosis is not a frequent finding in places where access to rapid diagnosis and proper management of condylar fracture is available ${ }^{[12]}$. Most of the patients were from rural areas with no easy access to dental surgical specialist which can explain the findings that are also in match with other comparable communities. Qudah et al in $2005^{[13]}$ reported trauma incidence in a group of Jordanian children to be $100 \%$. These results are comparable to other reports ${ }^{[14,15]}$.

The patient problem is not only the mastication and speech but also oral hygiene and the mandible growth if the patient is in growing age ${ }^{[16]}$. This is how patients were motivated in this study. Most of the patients were from rural areas so motivation is important to avoid neglection of follow-up.

The clinical diagnosis of cases of this study revealed limitation of mandibular movement, deviation of jaw, reduced ramus height and a lot of other jaw deformities. On the other hand, the radiographical evaluation revealed deformation in condyle, joint space obliteration, retruded mandibular position and other undergrowth features, a findings in match with other literatures ${ }^{[17-19]}$. 
Three dimensional CT evaluation is the most reliable imaging technique for assessment of cases

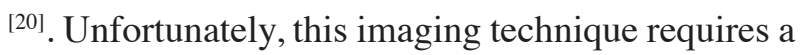
high dose of radiation which is not suitable for follow up if not justified. Also, when available, it may be out of the financial reach of certain socioeconomic level of citizens who cannot afford the cost. So that, in this study, CT is used in preoperative assessment and at 2 years followup as the good is more than harm when confirming the diagnosis, assessing the anatomical relations, help in surgical planning and as a followup record.

Gap arthroplasty is the surgical technique used for releasing the ankylotic mass by removal of a block of bone, leaving a gap between the ascending ramus and temporal bone. Also, different protocols can be used to prevent reankylosis and the correction of associated deformities should be always in mind ${ }^{[21-24]}$.

A balanced surgical intervention should be done as if the gap is too small, recurrence is more likely and on the other side if it is large, mandibular height is lost [9]. Surprisingly recurrence can develop, even after wide gap arthroplasty ${ }^{[25]}$. That's why in this study, conservative gap is the technique of choice following the steps of Babu et al ${ }^{[9]}$ who used a minimal gap of 5-8 $\mathrm{mm}$ with interposing temporalis muscle and reports no recurrence after follow-up of three years. In this study conservative vertical gap of 7-9 $\mathrm{mm}$ along the ankylotic mass was done aiming to preserve the vertical dimension of the ramus and to allow remodelling of the ramus to a condyle-like end so no need for reconstruction of the TMJ, a result that was proved by both postoperative clinical and radiographic follow up.

Interposition of material may allow less gap width but with morbidity of the donor site, which is against the aim of this work in utilizing the most conservative approach. Bhat et al ${ }^{[25]}$ compared the using and not using of interpositioning material (temporalis) and found a comparable satisfactory outcomes and recurrence rate. They also found that an intact temporalis muscle may have a protective effect on open-bite prevention. That's why conservative gap without interpositioning material was the chosen protocol.

The amount of postoperative mouth opening achieved was higher than that reported by others ${ }^{[9}$, 26]. There was evident improvement at subsequent follow-up visits which can be attributed to not only the narrow gap but also to the physiotherapy protocols that enhance remodeling of the stump of the ramus and allow some degree of neuromusculature re-education.

The results of the present study show the importance of immediate postoperative physiotherapy, a findings in match with other reports ${ }^{[1,14]}$.

The using of postoperative exercises aim to disrupt and prevent adhesions and allow normal muscle function for maintenance of intraoperative mouth opening and decreasing the possibility reankylosis. Although physiotherapy was painful, the results were not only preventing reankylosis but also the mouth opening was increased. Pain subsided after two weeks.

The limitations of this study include the small sample size which may be attributed to the nature of TMJ ankylosis as being not a common condition. The study also had a relatively short follow-up and a longer follow up is the future planning. Although they all had same diagnosis and were treated in the same way, patients were not followed until the completion of skeletal growth to study the effect of treatment in growth and facial appearance. Long term follow up for growth is also recommended.

\section{REFERENCES}

1. Bello, S.A., et al., Aetiology and presentation of ankylosis of the temporomandibular joint: report of 23 cases from Abuja, Nigeria. British Journal of Oral and Maxillofacial Surgery, 2012. 50(1): p. 80-84.

2. Long, X., et al., Preservation of disc for treatment of traumatic temporomandibular joint ankylosis. Journal of oral and maxillofacial surgery, 2005. 63(7): p. 897-902. 
3. Loveless, T.P., et al., Efficacy of temporomandibular joint ankylosis surgical treatment. Journal of Oral and Maxillofacial Surgery, 2010. 68(6): p. 1276-1282.

4. Zhi, K., et al., Management of temporomandibular joint ankylosis: 11 years' clinical experience. Oral Surgery, Oral Medicine, Oral Pathology, Oral Radiology, and Endodontology, 2009. 108(5): p. 687-692.

5. Elgazzar, R., et al., Treatment modalities of TMJ ankylosis: experience in Delta Nile, Egypt. International journal of oral and maxillofacial surgery, 2010. 39(4): p. 333-342.

6. Felstead, A.M. and P.J. Revington, Surgical management of temporomandibular joint ankylosis in ankylosing spondylitis. International journal of rheumatology, 2011.2011.

7. Maheshwari, S., et al., Biomechanics and orthodontic treatment protocol in maxillofacial distraction osteogenesis. National journal of maxillofacial surgery, 2011.2(2): p. 120.

8. Kaban LB, Bouchard C, Troulis MJ. A protocol for management of temporomandibular joint ankylosis in children. J Oral Maxillofac Surg 2009;67:1966-78.

9. Babu, L., et al., Is aggressive gap arthroplasty essential in the management of temporomandibular joint ankylosis?a prospective clinical study of 15 cases. British Journal of Oral and Maxillofacial Surgery, 2013. 51(6): p. 473-478.

10. Munro, I.R., Y.R. Chen, and B.Y. Park, Simultaneous total correction of temporomandibular ankylosis and facial asymmetry. Plastic and reconstructive surgery, 1986. 77(4): p. 517-529.

11. Kaban, L.B., C. Bouchard, and M.J. Troulis, A protocol for management of temporomandibular joint ankylosis in children. Journal of Oral and Maxillofacial Surgery, 2009. 67(9): p. 1966-1978.

12. Rishiraj, B. and L.R. McFadden, Treatment of temporomandibular joint ankylosis: a case report. Journal-Canadian Dental Association, 2001. 67(11): p. 659-663.

13. Qudah, M.A., M.A. Qudeimat, and J.Al-Maaita, Treatment of TMJ ankylosis in Jordanian children-a comparison of two surgical techniques. Journal of Cranio-Maxillofacial Surgery, 2005. 33(1): p. 30-36.

14. Matsuura, H., et al., The effect of gap arthroplasty on temporomandibular joint ankylosis: an experimental study. International journal of oral and maxillofacial surgery, 2001. 30(5): p. 431-437.

15. Kaban, L.B., D.H. Perrott, and K. Fisher, A protocol for management of temporomandibular joint ankylosis.
Journal of oral and maxillofacial surgery, 1990. 48(11): p. 1145-1151.

16. Movahed, R. and L.G. Mercuri, Management of temporomandibular joint ankylosis. Oral and Maxillofacial Surgery Clinics, 2015. 27(1): p. 27-35.

17. Zhu, Y., et al., Cephalometric analysis on patients with TMJ ankylosis in different ages. Shanghai kou qiang yi xue= Shanghai journal of stomatology, 2000. 9(3): p. 132-134.

18. Ko, E., et al., Cephalometric craniofacial characteristics in patients with temporomandibular joint ankylosis. Chang Gung medical journal, 2005. 28(7): p. 456

19. Shashikiran, N., et al., Management of temporo-mandibular joint ankylosis in growing children. Journal of Indian Society of Pedodontics and Preventive Dentistry, 2005. 23(1): p. 35.

20. De Bont, L., et al., Computed tomography in differential diagnosis of temporomandibular joint disorders. International journal of oral and maxillofacial surgery, 1993. 22(4): p. 200-209.

21. Mohan, M.C., et al., Reconstruction of condyle following surgical correction of temporomandibular joint ankylosis: current concepts and considerations for the future. Nitte University Journal of Health Science, 2014. 4(2): p. 39.

22. Abbe, R., An operation for the relief of ankylosis of the temporomaxillary joint by exsection of the neck of the condyle of the lower jaw. NY Med J, 1880. 81: p. 362-366.

23. Braimah, R., et al., Clinical experience in managing temporomandibular joint ankylosis: five-year appraisal in a Nigerian subpopulation. Journal of the Korean Association of Oral and Maxillofacial Surgeons, 2018. 44(3): p. 112-119.

24. Al-Moraissi, E., et al., A systematic review and metaanalysis of the clinical outcomes for various surgical modalities in the management of temporomandibular joint ankylosis. International journal of oral and maxillofacial surgery, 2015. 44(4): p. 470-482.

25. BHATT, Krushna, et al., Functional outcomes of gap and interposition arthroplasty in the treatment of temporomandibular joint ankylosis. Journal of Oral and Maxillofacial Surgery, 2014. 72(12): p. 2434-2439.

26. Roychoudhury, A., H. Parkash, and A. Trikha, Functional restoration by gap arthroplasty in temporomandibular joint ankylosis: a report of 50 cases. Oral Surgery, Oral Medicine, Oral Pathology, Oral Radiology, and Endodontology, 1999. 87(2): p. 166-169. 